\title{
An EOQ Model for Phase Inventory with Induced Demand and Periodic Cycle Time
}

\author{
Sujit Kumar De, ${ }^{1}$ Shib Sankar Sana, ${ }^{2}$ and Adrijit Goswami ${ }^{3}$ \\ ${ }^{1}$ Department of Mathematics, Midnapore College, Vidyasagar University, Medinipur, West Bengal 721101, India \\ ${ }^{2}$ Department of Mathematics, Bhangar Mahavidyalaya, South 24 Parganas, West Bengal 743502, India \\ ${ }^{3}$ Department of Mathematics, Indian Institute of Technology, Kharagpur 721302, India
}

Correspondence should be addressed to Shib Sankar Sana; shib_sankar@yahoo.com

Received 27 May 2014; Revised 17 August 2014; Accepted 25 August 2014; Published 16 September 2014

Academic Editor: Eleonora Bottani

Copyright (C) 2014 Sujit Kumar De et al. This is an open access article distributed under the Creative Commons Attribution License, which permits unrestricted use, distribution, and reproduction in any medium, provided the original work is properly cited.

\begin{abstract}
This paper deals with a stock flow of an inventory problem over induced demand. The inventory is consumed through "core customer" or chain marketing system in an induced environment (inductance) to exhaust all the items of the stock inventory in an indefinite time. The demand rate is depicted due to induced factor which is generated from the same inventory presented nearby. The inventory cycle time is split into several periodic times due to oscillatory feature of the inventory which is called phase inventory. Considering uniform demand, this cycle time splits into two basic parts, namely, "first shift" (phase) and "second shift" (phase). Since the process dampens over time, so the whole inventory will exhaust after few periods. A cost function consisted of inventory cost, setup cost, and loss for induced items is minimized to obtain optimal order quantity and replenishment time. The multivariate lagrange interpolation (MLI) over the average values of the postsensitivity analysis is developed here. Finally, graphical illustrations are made to justify the model.
\end{abstract}

\section{Introduction}

Recently, a great evolution has been made in the traditional EOQ model. Through its process, a survey work of the industrial system over Harris's work [1] in the last century has been made by Andriolo et al. [2]. A capacitated lot sizing problem for coordination of transportation services is developed by Bruno et al. [3]. A review of consignment stocking policy under advertising, pricing, and bargaining strategies is also discussed by Sarker [4]. Moreover, several articles of the inventory in supply chain [5-11] have suggested that discounting on sales price is the emergent way to exhaust all items from the inventory in due course of time. Bozorgi et al. [12] develop a new model where the inventory moves with environmental temperature, controlled by emission function. Sodhi et al. [13] state the bullwhip effect on maintenance repair and overhaul (MRO) customers. Analysis of batch ordering inventory for infinite horizon with capacity constraints is studied by Yang et al. [14]. Coelho and Laporte [15] derive an inventory routing problem
(IRP) for the inequalities, based on demand and available capacities. Also, Avinadav et al. [16] are able to develop a multiplicative demand function relating to age and price effect, whereas Chand and Sethi [17] made an analysis for dynamic and stationary demand in multiperiod lot sizing problem for both finite and infinite time horizons in the same time. Keskin and Capar [18] describe a decision support system model on the basis of the utility of three pillars of supply chain management, namely, location-transportationinventory (LTI). Gambini et al. [19] investigate an EOQ model where stocking charge is independent of time and the stock flow down depends on its level only. Dolan [20] observes the effect of quantity discount on the joint EOQ model and suggests an optimal strategy. Monahan [21] develops a quantity discount model from the perspective of the seller with the constraints which are imposed to ensure sufficient benefit to the buyer. A continuous discount pricing model is explained by Dave et al. [22]. Tripathy and Pattanaik [23] analyze a fuzzy entropic model with stock dependent demand for perishable items. Syed and Aziz [24] develop a fuzzy price 
break model also. A model with perishable asset revenue management is studied by Weatherford and Bodily [25]. All of the above authors assure that the discounting on sales prices of the items in stock increases selling and it would be continued until the whole inventory is exhausted.

However, the present world economy is very much crucial and the uncertainty in marketing system is increasing day by day. In this situation, nobody can assure that salesman's commodities would be purchased just after the opening of his/her shop. In our observations, such facts occur mainly due to two basic reasons.

(a) Firstly, the purchasing capacity of the common people is decreasing day by day due to profit making business of multinational companies (MNC) throughout the world, especially in third world countries. As a result, a huge number of products are being piled as time progresses. This phenomenon is commonly known as "crisis of capitalism."

(b) The second one is the high competition among various companies and few reputations of them. A reputed company attracts customers easily rather than nonreputed ones as they have their own "core customer circle" (see Definition 2). These core customers may be influenced by other MNC at the time of purchasing the goods just to have separate tastes and feelings of new emerging items of the market. On the other hand, for a newly set-up shop/company, naturally they have neither "reputation" nor "core customer circle" but they have to struggle through this system. Such types of companies/salesmen begin to start their business with few peer (core, friend-relative circle) customers. The customers (core/noncore) who are influenced or motivated by the advertisement through the printed/digital medias/sales teams are called "floating" or "surface" (see Definition 1) customers.

Behavioral study of the customers indicates that the floating customers would not always purchase goods at discounted price but to oppose many cases although they were the surface customers in few days ago. The general motives of the customers are "purchase quality goods at fair price"; their views on discounted items are usually as follows: "unfair, bad quality or very near to outdated time bar." So, a negative motivation arises through the announcement "discounted items." Hence, the unsold items are rotated periodically for an indefinite time.

The consumption rate of common assets like mobile phone is increasing day by day. At the same time, critical risks and challenges are observed among the various mobile service providers. The main challenges are coming from the frequent change of SIM (subscriber identity module) cards among the customers. For this reason, several companies are offering price discount to capture more customers in favor of them. In this way, for instance, Vodafone Company has captured India's largest telephone company Bharat
Sanchar Nigam Limited, BSNL's market. Likewise, Airtel, MTS, DOCOMO, UNINOR, and so forth have captured partially Vodafone's market. Under the circumstances, a SIM card seller of any company, after purchasing (manufacturing) a certain amount of items, will have to wait a long time until all the items are exhausted from its own inventory. On the other hand, the demand of assets/goods like car, motor bike, watch, ceiling fan, almirah, utensils, soaps, and so forth in a particular enterprise may fall under this consideration. Thus, the lingering time to exhaust all items is basically due to the presence of another shop (inventory setup that offers price discount or not) in which every customer (core/noncore) has multiple options to choose their better service provider. Now, we define floating customers as induced customers in which the products are not out-flowed from the inventory system for opposed demand due to "induced factor," and the inventory relating to this system is termed as phase or periodic inventory. Therefore, the behavior of the inventory function is quite similar to the discharging process in a capacitor of an electrical circuit through inductance. In this literature, several attempts in trade economics have been made by several researchers. Bryant's [26] thermodynamic approach to the financial economics is quite familiar. Similarly, a classical thermodynamic and general equilibrium theory has been developed by Smith and Foley [27] and the role of money is developed by Soddy [28].

In our present study, we made an attempt for analyzing an inventory model under electrical circuit theory (ECT). Here, the order quantity is independent of time, so we have studied the MLI function over the postsensitivity analysis of the model to make a final conclusion.

\section{Assumptions and Notations}

The following notations and assumptions are used to develop the model.

Assumptions

(1) The inventory starts at the beginning (time $t=0$ at the time phase difference) with maximum on-hand inventory and depletes through natural system. After adjusting the demand of the customers at two phases of each cycle time, it reaches zero level at the end of the cycle time.

(2) The time horizon is infinite (days).

(3) Shortages are not allowed.

(4) Demand rate is a time dependent phase inventory function.

(5) Holding cost is uniform over the cycle time.

(6) Inductance starts at the linear time phase difference and ends at the time of the twice multiple of linear time phase difference in the first shift of the last period.

(7) Conducting factor is low. 
(8) Items are similar which are stored in the shops nearby.

(9) Total number of customers for particular types of product is fixed.

(10) Expiry date of the items is indefinite.

\section{Notation}

$q_{0}$ : The maximum order quantity per cycle

$D(t)$ : Instantaneous demand at time $t$ in $\left(0, T_{1}\right)$

$q(t)$ : The instantaneous inventory at time $t$ in $\left(0, T_{1}\right)$

$\theta$ : The damping factor

$x$ : Linear time phase difference

$h$ : Inventory holding cost per unit item per time unit (\$)

$b$ : Set-up cost per cycle (\$)

$c_{0}$ : Cost of induced factor per unit induced demand item (\$)

$m$ : The number of days per cycle, a positive integer

$T_{1}$ : Replenishment time in days

$C$ : Capacity factor

$L:$ Induced factor

$R$ : Resisting index

$Z$ : Average inventory cost per period (day) (\$).

\section{Formulation of the Model}

3.1. Stock Flow Inventory Model under Inductance. Let us consider an inventory chain having no external supply of goods. The circuit has some resisting factor $R$ due to the weak presence of core customers and induced factor $(L)$ due to the presence of other shops nearby. In this system, a seller after purchasing a maximum order quantity began to sale through the core customers with the presence of floating customers. The inventory is not exhausted after a long elapsed time whenever the core customers are absent in the system (see (3)-(6)), but the system dampens slowly the process and all the items would be exhausted few days later (see Figure 1).

Definition 1 (floating or surface customers (induced factor)). Such customers do not have any faith or belief in any kind of branded company. They can influence core customers and are influenced by the upcoming advertisement of several companies. Therefore, their presence in the inventory system can create a harmonious induced factor $L$ or threat in the system itself. It is defined as a percent of customers who do not purchase items due to the influences of other customers/shops within a certain interval of time.

Definition 2 (core customers (conducting factor)). Every inventory circuit has specific challenges or limitations (resisting factor $R$ ). Conversely, every inventory circuit (seller's network) has its own peer/friend circle or chain marketing system (however small it may be) who will purchase goods/commodities from his (her) own system. They are

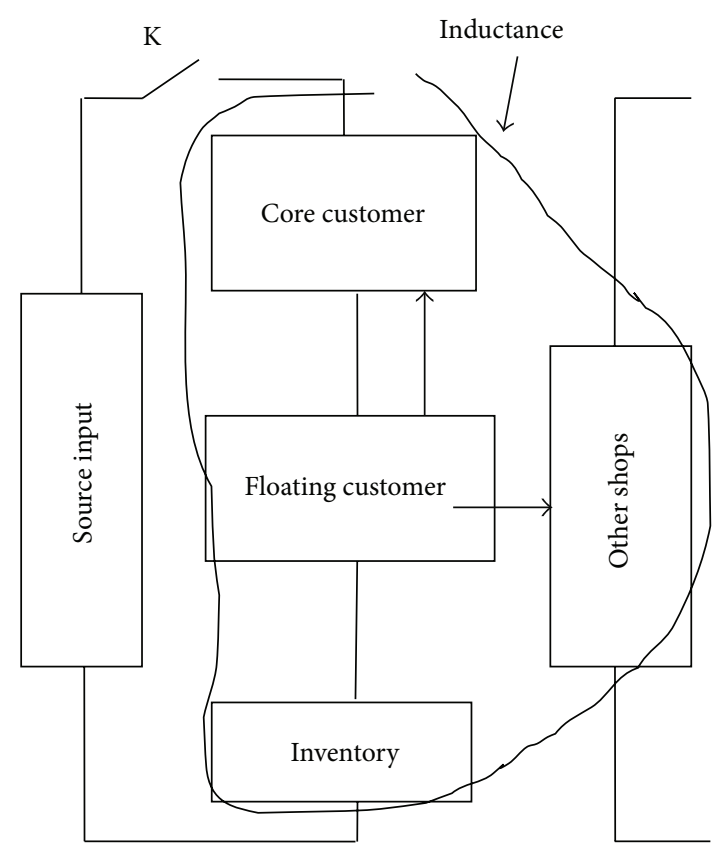

FIgURE 1: Inventory system.

called core customers. The resisting factor $R$ can be defined as a percent of customers who do not like to purchase the items from the particular inventory system due to dissatisfaction of service providers, lack of goodwill and misconduct from the selling personnel, and so forth. Therefore, the $(100-$ $R) \%$ of satisfied customers acts as a conducting factor of the inventory system.

Definition 3 (induced items). It is defined as the amount of goods that are not sold due to induced factor. If $D(t)$ is the instantaneous demand rate at time $t$, then the total amount of inventory that is unsold due to induced factor is given by

$$
L \frac{d D(t)}{d t}=\frac{1}{C w_{0}^{2}} \frac{d^{2} q}{d t^{2}}, \quad \text { where } D(t)=-\frac{d q}{d t},
$$

where $q$ is the instantaneous inventory at time $t$.

Definition 4 (capacity factor). It is used to define the degree in which the items $q_{0}$ are accommodating in the shop for sale in the indefinite time horizon. It is denoted by $C$ and is defined by resisting index $R$, induced factor $L$, and the damping factor of the system (see Table 1 ).

Definition 5 (shift frequency/time phase). Here, the frequency is the number of cycles (periods) that occurred in a particular time. Let, at least, one period occur in a day; more than one day may correspond to a single period also. It is defined as the total number of turnovers per day. We may assume that the inventory curve completes at least one cycle (reach a similar phase point) in a day and gives two 
TABLE 1: Summary of measurement.

\begin{tabular}{|c|c|c|c|c|c|c|c|}
\hline Parameters & $q$ & $D(t)$ & C & $L$ & $x$ & $T$ & $\theta<1$ \\
\hline Inventory system & $\frac{q_{0} e^{-2 \pi \theta t} \sin w(t+x)}{\sqrt{1-\theta^{2}}}$ & $-\frac{d q}{d t}$ & $\frac{\theta}{\pi}\left(\frac{1}{R}\right)$ & $\frac{1}{4 \pi^{2} C}$ & $\frac{1}{8 \sqrt{1-\theta^{2}}}$ & $\frac{1}{\sqrt{1-\theta^{2}}}$ & $\frac{R}{2} \sqrt{\frac{C}{L}}$ \\
\hline
\end{tabular}

different phase intervals in which the curve gets positive (first shift) and negative values (second shift) successively (in a day, repeated phase time is not possible in general).

So for a complete oscillation, the inventory curve will take just one day; hence, its frequency is one; that is, $f_{0}=1$. Thus to have circular frequency we take

$$
\begin{aligned}
& w_{0}=2 \pi f_{0}=2 \pi \times 1=2 \pi, \\
& w=w_{0} \sqrt{1-\theta^{2}}=2 \pi \sqrt{1-\theta^{2}} .
\end{aligned}
$$

Definition 6 (periodic time $T$ ). It is defined as the time (day) required to complete one closed path (period) of the inventory curve and is denoted by

$$
T=\frac{2 \pi}{w}=\frac{1}{\sqrt{1-\theta^{2}}} .
$$

If the inventory circuit has no core customers, then the periodic time is $T_{0} ; w \approx w_{0}$ and $T \approx T_{0}$. And

$$
T>T_{0} \quad w<w_{0} \quad \forall \theta<1
$$

3.2. Multivariate Lagrange Interpolation (MLI). Let $f=$ $f\left(X_{1}, X_{2}, X_{3}, \ldots, X_{m}\right)$ be an $m$-variable multinomial function of degree $n$. Since there are $\left(\begin{array}{c}n+m \\ n\end{array}\right)=\rho$ terms in $f$, it is a necessary condition that we have $\rho$ distinct points $\left(x_{1, i}, x_{2, i}, x_{3, i}, \ldots, x_{m, i}, f_{i}\right) \in R^{m+1}, 1 \leq i \leq \rho, f_{i}=$ $f\left(x_{1, i}, x_{2, i}, x_{3, i}, \ldots, x_{m, i}\right)$, for $f$ to be uniquely defined. In other words, $f\left(X_{1}, X_{2}, X_{3}, \ldots, X_{m}\right)=\sum_{e_{i}, 1 \leq n} \alpha_{e_{i}} X^{e_{i}}$, where the $\alpha_{e_{i}}$ are the coefficients in $f . X=\left(X_{1}, X_{2}, X_{3}, \ldots, X_{m}\right)$ is the $m$-tuple of independent variables of $f \cdot e_{i}=$ $\left(e_{1 i}, e_{2 i}, \ldots, e_{m i}\right)$ is an exponent vector with nonnegative integer entries, consisting of an ordered partition of an integer between 0 and $n$ inclusively. $e_{i} \cdot 1=\sum_{j=1}^{m} e_{j i}$ is the usual vector dot product; $X^{e_{i}}=\prod_{j=1}^{m} X_{j}^{e_{j i}}$. Now we wish to write $f$ in the form $\sum_{i=1}^{\rho} f_{i} l_{i}(X)$, where $l_{i}(X)$ is a multinomial function in the independent variables $\left(X_{1}, X_{2}, X_{3}, \ldots, X_{m}\right)$ with the property that when $X$ is equal to $i$ th data value or $X=$ $x_{i}\left(\left(X_{1}, X_{2}, X_{3}, \ldots, X_{m}\right)=\left(x_{1, i}, x_{2, i}, x_{3, i}, \ldots, x_{m, i}, f_{i}\right)\right)$, then $l_{i}\left(x_{i}\right)=1$ and $l_{j}\left(x_{i}\right)=0(j \neq i)$. Let us consider the system of linear equations

$$
f_{i}=\sum_{e_{i}, 1 \leq n} \alpha_{e_{i}} x_{i}^{e_{i}}, \quad \text { where } 1 \leq i \leq \rho
$$

From this system, we construct the sample matrix

$$
M=\left[x_{i}^{e_{i}}\right]=\left(\begin{array}{ccc}
x_{1}^{e_{1}} & \cdots & x_{1}^{e_{\rho}} \\
x_{i}^{e_{1}} & \cdots & x_{i}^{e_{\rho}} \\
x_{\rho}^{e_{1}} & \cdots & x_{\rho}^{e_{\rho}}
\end{array}\right)
$$

Assuming $\operatorname{det}(M) \neq 0$, we could solve for the coefficients $\alpha_{i}$ in $f$ by inverting $M$.

If $M$ is singular, then the coefficients of $f$ are not uniquely determined, characterizing the geometric configuration of the $\rho$ points so that $\operatorname{det}(M)=0$ appears to be an intricate problem.

Let $\Delta=\operatorname{det}(M) \neq 0$. Substituting $x_{j}=X$ in $M$, we have

$$
\begin{gathered}
M_{j}(X)=\left(\begin{array}{ccc}
x_{1}^{e_{1}} & \cdots & x_{1}^{e_{\rho}} \\
X^{e_{1}} & \cdots & X^{e_{\rho}} \\
x_{\rho}^{e_{1}} & \cdots & x_{\rho}^{e_{\rho}}
\end{array}\right) \longleftarrow j \text { th row } \\
\Delta_{j}(\mathrm{X})=\operatorname{det}\left(M_{j}(X)\right) .
\end{gathered}
$$

Next, substituting $X=x_{i}$ in $M_{j}(X),(i \neq j)$, we have the following matrix $\left(M_{j}\right)_{i}$ :

$$
\left(M_{j}\right)_{i}=\left(\begin{array}{ccc}
x_{1}^{e_{1}} & \ldots & x_{1}^{e_{\rho}} \\
x_{i}^{e_{1}} & \cdots & x_{e_{\rho}}^{e_{\rho}} \\
x_{i}^{e_{1}} & \cdots & x_{i}^{e_{\rho}} \\
x_{\rho}^{e_{1}} & \cdots & x_{\rho}^{e_{\rho}}
\end{array}\right) \longleftarrow i \text { th row }
$$

Here, the $i$ th row appears twice in $\left(M_{j}\right)_{i}$ that means $\operatorname{det}\left(M_{j}\right)_{i}=0$. In other words, $\Delta_{j}\left(x_{i}\right)=0(i \neq j)$ at $X=x_{i}$. By construction, $X=x_{i} \Rightarrow \Delta_{i}(\mathrm{X})=\Delta$ that provides $l_{i}(X)=\Delta_{i}(\mathrm{X}) / \Delta$. Therefore,

$$
f=\sum_{i=1}^{\rho} f_{i} \frac{\Delta_{i}(\mathrm{X})}{\Delta} .
$$

Applying the above definitions and interpolation function, we will discuss our proposed model as follows.

Let the inventory start at a time phase point $x($ at $t=0)$ and exhaust at the first shift of the last day after covering $m$ full days of the time horizon $\left(0, T_{1}\right)$. The induction starts after $x$ time later and ends at the $2 x$ time of the first shift of the last day. Then, the governing differential equation is

$$
L \frac{d^{2} q}{d t^{2}}=R D(t)+\frac{1}{C} \int_{-\infty}^{t} D(\tau) d \tau
$$

with $q=q_{0}$ and $d q / d t=0$ at time $t=0$. 
Now, substituting $d q / d t=-D(t)$ in the above, we have

$$
L \frac{d^{2} q}{d t^{2}}+R \frac{d q}{d t}+\frac{q}{C}=0
$$

Now, if no external supply is supplied to the chain, then (11) reduces to

$$
\frac{d^{2} q}{d t^{2}}+2 \theta w_{0} \frac{d q}{d t}+w_{0}^{2} q=0
$$

where

$$
\begin{gathered}
w_{0}=\frac{1}{\sqrt{L C}}, \\
\theta=\frac{R}{2 w_{0} L}=\frac{R}{2} \sqrt{\frac{C}{L}}
\end{gathered}
$$

is called the damping factor, and the initial conditions are given by

$$
q=q_{0}, \quad \frac{d q}{d t}=0 \quad \text { for } t=0 .
$$

The solution of (12) for $\theta<1$ is given by

$$
q(t)=\frac{q_{0} e^{-\theta w_{0} t} \operatorname{Sin}(w t+\varphi)}{\sqrt{1-\theta^{2}}}
$$

where

$$
\begin{array}{r}
w=w_{0} \sqrt{1-\theta^{2}}, \quad \varphi=\frac{2 \pi x}{\delta}=w x \\
{[\text { see }(\text { A.1) }] .}
\end{array}
$$

Here, $\varphi$ is the phase difference, $x$ is the linear distance, $\delta$ is the web length of the oscillatory curve, and the period of oscillation is given by

$$
T=\frac{2 \pi}{w}=\frac{2 \pi}{w_{0} \sqrt{1-\theta^{2}}} .
$$

Also,

$$
\operatorname{Tan} w x=\frac{\sqrt{1-\theta^{2}}}{\theta} \Longrightarrow \frac{\operatorname{Sin} w x}{\sqrt{1-\theta^{2}}}=\frac{\operatorname{Cos} w x}{\theta}=1 .
$$

Again from (19),

$$
w x=\frac{\pi}{4} \Longrightarrow w_{0} x \sqrt{1-\theta^{2}}=\frac{\pi}{4} \Longrightarrow x=\frac{\pi}{4 w_{0} \sqrt{1-\theta^{2}}} .
$$

Therefore, the on-inventory is as follows:

$$
q(t)=\frac{q_{0} e^{-2 \pi \theta t} \operatorname{Sin} w(t+x)}{\sqrt{1-\theta^{2}}} \text { for } 0 \leq t \leq T_{1} .
$$

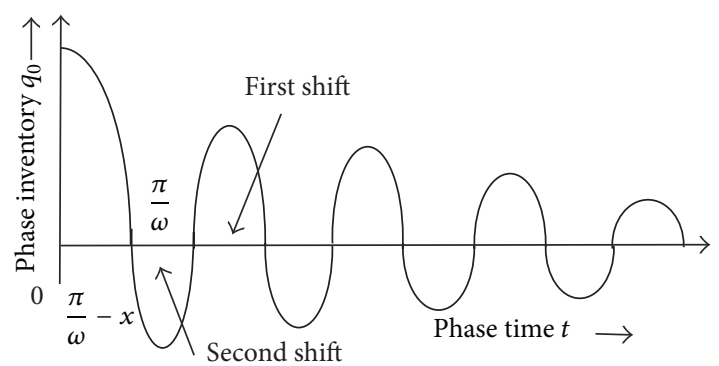

FIgURE 2: Phase inventory model.

We see (Figure 2) that the time domain of the first shift $[q(t)$ is $+\mathrm{ve}]$ and the second shift $[(q(t)$ is $-\mathrm{ve}]$ is as follows:

First shift: $t \in\left[0, \frac{\pi}{w}-x\right] \cup\left[\frac{2 r \pi}{w}-x, \frac{(2 r+1) \pi}{w}-x\right]$, $r=1,2,3, \ldots, m$

Second shift: $t \in\left[\frac{(2 r-1) \pi}{w}-x, \frac{2 r \pi}{w}-x\right]$,

$$
r=1,2,3, \ldots, m
$$

$$
T_{1}=\frac{8 m+3}{8 \sqrt{1-\theta^{2}}}
$$

Now, the inventory holding cost for the first shift (phase) per cycle is

$$
\begin{aligned}
\mathrm{HCD}= & h\left\{\int_{0}^{t_{1}} q(t) d t+\int_{t_{2}}^{t_{3}} q(t) d t\right. \\
& \left.\quad+\int_{t_{4}}^{t_{5}} q(t) d t+\cdots \int_{t_{2 m}}^{t_{2 m+1}} q(t) d t\right\} \\
= & h \int_{0}^{\pi / w-x} q(t) d t+h \sum_{r=1}^{m} \int_{2 r \pi / w-x}^{(2 r+1) \pi / w-x} q(t) d t \\
= & \frac{h q_{0}}{2 \pi}\left[2 \theta+e^{2 \pi \theta x-2 \theta \pi^{2} / w}\right]+\frac{h q_{0} e^{2 \pi \theta x-2 \theta \pi^{2} / w}}{2 \pi} \\
& \times\left[\frac{1-e^{-4 m \theta \pi^{2} / w}}{e^{2 \theta \pi^{2} / w}-1}\right]
\end{aligned}
$$

[see (A.7)] 
The inventory holding cost for the second shift (phase) per cycle is given by

$$
\begin{aligned}
\mathrm{HCN}= & -h\left\{\int_{t_{1}}^{t_{2}} q(t) d t+\int_{t_{3}}^{t_{4}} q(t) d t\right. \\
& \left.\quad+\int_{t_{5}}^{t_{6}} q(t) d t+\cdots \int_{t_{2 m-1}}^{t_{2 m}} q(t) d t\right\} \\
= & -h \sum_{r=1}^{m} \int_{(2 r-1) \pi / w-x}^{2 r \pi / w-x} q(t) d t \\
= & \frac{h q_{0} e^{2 \pi \theta x}}{2 \pi}\left[\frac{1-e^{-4 m \theta \pi^{2} / w}}{e^{2 \theta \pi^{2} / w}-1}\right] .
\end{aligned}
$$

Now using (14), the loss per cycle due to the induced factor is

$$
\begin{aligned}
\text { LOI } & =c_{0} L \int_{0}^{T_{1}} \frac{d^{2} q(t)}{d t^{2}} d t \\
& =\frac{8 c_{0} q_{0} L \pi^{3}}{w} \int_{0}^{T_{1}} e^{-2 \pi \theta t} \operatorname{Sin} w(t-x) d t \\
& =\frac{2 \pi c_{0} q_{0}}{C w}\left(\sum_{r=0}^{m-1} \int_{2 r \pi / w+x}^{(2 r+1) \pi / w+x} e^{-2 \pi \theta t} \operatorname{Sin} w(t-x) d t\right. \\
& =\frac{\left.-\sum_{r=1}^{m} \int_{(2 r-1) \pi / w+x}^{2 r \pi / w+x} e^{-2 \pi \theta t} e^{-2 \pi \theta x} \operatorname{Sin} w(t-x) d t\right)}{2 \pi C\left(1-e^{\left.-2 \theta \pi^{2} / w\right)}\left(1+e^{-2 \theta \pi^{2} / w}\right)\left(1-e^{-4 m \theta \pi^{2} / w}\right)\right.}
\end{aligned}
$$

Now, using (23), (24), and (25), the average profit per day is given by

$$
\begin{aligned}
Z= & \text { Holding cost (first \& Second phase) } \\
& + \text { Loss for induced items + set up cost }] \\
& \times(\text { cycle time })^{-1} \\
= & \left\{\frac{h q_{0}}{2 \pi}\left[2 \theta+e^{2 \pi \theta x-2 \theta \pi^{2} / w}\right]\right. \\
& +\frac{h q_{0} e^{2 \pi \theta x}}{2 \pi}\left(1+e^{-2 \theta \pi^{2} / w}\right)\left[\frac{1-e^{-4 m \theta \pi^{2} / w}}{e^{2 \theta \pi^{2} / w}-1}\right] \\
& +\frac{c_{0} q_{0} e^{-2 \pi \theta x}}{2 \pi C\left(e^{2 \theta \pi^{2} / w}-1\right)}\left(1+e^{2 \theta \pi^{2} / w}\right) \\
& \left.\times\left(1-e^{-4 m \theta \pi^{2} / w}\right)+b\right\} \frac{1}{T_{1}} .
\end{aligned}
$$

Now using Table 1, our problem reduces to

$$
\text { Minimize } Z=\frac{4 \sqrt{4 L-C R^{2}}}{(8 m+3) L^{2}}\left[F_{1}+F_{2}+F_{3}+b L \sqrt{L}\right] \text {, }
$$

where

$$
\begin{aligned}
& F_{1}=h q_{0}\left(C L^{2}+R C L \sqrt{L}-\frac{3}{16} R L \sqrt{C}+\frac{9}{512} R^{2} \sqrt{C}\right. \\
& \left.-\frac{3}{128} R^{3} C \sqrt{C}\right) \\
& F_{2}=2 m h q_{0}\left(2 L^{2} \sqrt{C}-\frac{1}{8} R L \sqrt{C}+\frac{9}{512} R^{2} L C \sqrt{C}\right. \\
& \left.-\frac{1}{64} R^{3} C \sqrt{C}\right) \\
& \times \frac{C R^{2}+8 L-2 m R}{C R^{2}+8 L+R}, \\
& F_{3}=\frac{2 m c_{0} q_{0}}{C}\left(2 L^{2} \sqrt{C}+\frac{1}{8} R L \sqrt{C}+\frac{1}{64} R^{2} \sqrt{C}\right. \\
& \left.+\frac{1}{64} R^{3} C \sqrt{C}\right) \\
& \times \frac{C R^{2}+8 L-2 m R}{C R^{2}+8 L+R} \\
& \text { subject to the conditions } T_{1}=\frac{8 m+3}{8 \sqrt{1-\theta^{2}}} \\
& e^{-R(8 m+3) / 8 \sqrt{4 L^{2}-L C R^{2}}}=0 \\
& R^{2} C<4 L \\
& \theta=\frac{R}{2} \sqrt{\frac{C}{L}} .
\end{aligned}
$$

Particular Cases: Since the model is developed with the specific cases.

(1) Cases of Optimality

Case I: when induced factor $L$ is fixed and the resisting index $R$ and conducting factor $C$ vary.

Case II: when resisting index $R$ is fixed and the induced factor $L$ and conducting factor $C$ vary.

Case III: when conducting factor $C$ is fixed and the resisting index $R$ and induced factor $L$ vary.

Case IV: when conducting factor $C$ is fixed and the resisting index $R$ and induced factor $L$ vary.

All measurements with different parameters are summarized in Table 1. 
TABLE 2: Solution of $R-L-C$ model for $L=20 \%$.

\begin{tabular}{|c|c|c|c|c|c|c|}
\hline$m$ & $T$ & $T_{1}$ & $R(\%)$ & $C$ & $\theta$ & $Z$ \\
\hline 1 & - & - & - & - & - & - \\
\hline 2 & 5.70 & 13.54 & 43 & 4.26 & 0.984 & 1502.44 \\
\hline 3 & 3.52 & 11.88 & 39 & 4.85 & 0.959 & 1229.14 \\
\hline 4 & 4.58 & 20.03 & 30 & 8.74 & 0.976 & $1207.94^{*}$ \\
\hline 5 & 4.32 & 23.24 & 24 & 13.63 & 0.973 & 1524.86 \\
\hline 6 & 4.47 & 28.47 & 20 & 19.65 & 0.975 & 1713.06 \\
\hline 7 & 1.27 & 9.36 & 14 & 16.41 & 0.616 & 4000.00 \\
\hline
\end{tabular}

TABLE 3: Solution of $R-L-C$ model for $R=20 \%$.

\begin{tabular}{|c|c|c|c|c|c|c|}
\hline$m$ & $T$ & $T_{1}$ & $L(\%)$ & C & $\theta$ & $Z$ \\
\hline 1 & 3.65 & 5.02 & 03 & 3.16 & 0.962 & $747.76^{*}$ \\
\hline 2 & 4.19 & 9.94 & 07 & 6.41 & 0.971 & 1032.51 \\
\hline 3 & 4.22 & 14.24 & 10 & 9.62 & 0.971 & 1285.43 \\
\hline 4 & 4.43 & 19.39 & 14 & 12.87 & 0.974 & 1418.98 \\
\hline 5 & 4.36 & 23.45 & 17 & 16.07 & 0.973 & 1601.08 \\
\hline 6 & 4.70 & 29.94 & 20 & 19.39 & 0.977 & 1623.87 \\
\hline 7 & 4.51 & 33.28 & 24 & 22.56 & 0.975 & 1807.61 \\
\hline 8 & 1.38 & 11.58 & 32 & 15.41 & 0.691 & 4000.00 \\
\hline
\end{tabular}

\section{Numerical Example}

We have considered the values of the parameters in appropriate units as follows.

Example 1. Let the values of parameters for Case I be $h=8$, $b=200, c_{0}=5$, and $q_{0}=500$. Then, we have the result (see Table 2).

Example 2. We consider the values of parameters for Case II in appropriate units as follows: $h=8, b=200, c_{0}=5$, and $q_{0}=500$. Then, we have the result (see Table 3 ).

Example 3. For Case III, let $h=8, b=200, c_{0}=5$, and $q_{0}=500$. Then, the result (see Table 4 ) is obtained.

Example 4. In Case IV, we consider $h=8, b=200, c_{0}=5$, and $q_{0}=500$. Then, we have the result (see Table 5).

4.1. Discussion of Tables 2-5. From Table 2, we see that if we take $20 \%$ induced factor over the whole time period, then the minimum average cost is $\$ 1207.94$ at total conducting factor 8.74 and resisting index $30 \%$, exhausting all the items within 20.03 days. In Table 3 , at $20 \%$ resisting index, the average minimum cost decreases to $\$ 747.76$ for total low induced factor $3 \%$ and the total conducting factor 3.16, exhausting all the items within 5.02 days. Table 4 shows that if the total conducting factor is 15 , then the average minimum cost comes down to $\$ 355.43$ for the total induced factor $0.12 \%$ and the resisting index $0.08 \%$, exhausting all the items within 6.38 days. However, Table 5 shows that the minimum average cost is $\$ 303.74$ at very high resisting factor (69.05) and average induced factor (11.80) for negligible conducting factor (0.009), exhausting all the items within 5.06 days.

\section{Sensitivity Analysis}

Considering $h=8, b=200, c_{0}=5, q_{0}=500$, and $C=$ 15 with appropriate units in the model and taking the range of variations of the parameters $\left\{h, c_{0}, q_{0}, C, b\right\}$ from $-50 \%$ to $+50 \%$ we have obtained optimal Table 6 .

5.1. Implication to Introduce Multivariate Lagrange Interpolation $(M L I)$. In the sensitivity analysis table, we usually see the maximum profit/minimum cost of the model for specific change of a particular parameter. This kind of result is quite limited to its local limitations. However, to have a global impact of the model we require a general objective function for the sensitivities of the whole parameters imparting in the system. But to increase our level of confidence, we need to know the range of the average cost function, the essential parts of any kind of inventory management.

From the solution Tables $2-5$, it is observed that the average cost attains its minimum value at the fixed values of conducting factor $C$. As a result, we have considered the sensitivity analysis of $C=15$ and we have constructed the MLI polynomial function in this case also.

First we construct the MLI for the six datasets $\left(\bar{L}, \overline{T_{1}}, \bar{Z}\right)$ that are shown in Table 7. 
TABLE 4: Solution of $R-L-C$ model for $C=15$.

\begin{tabular}{|c|c|c|c|c|c|c|}
\hline$m$ & $T$ & $T_{1}$ & $L(\%)$ & $R(\%)$ & $\theta$ & $Z$ \\
\hline 1 & 3.80 & 5.23 & 0.70 & 4 & 0.965 & 1245.61 \\
\hline 2 & 7.56 & 17.95 & 10 & 16 & 0.991 & 2339.02 \\
\hline 3 & 7.30 & 24.63 & 19 & 22 & 0.990 & 2710.98 \\
\hline 4 & 3.78 & 16.54 & 12 & 17 & 0.964 & 1743.59 \\
\hline 5 & 4.44 & 23.85 & 18 & 21 & 0.974 & 1537.77 \\
\hline 6 & 1.00 & 6.38 & 0.12 & 0.08 & 0.045 & $355.43^{*}$ \\
\hline 7 & 4.89 & 36.07 & 36 & 30 & 0.979 & 1446.49 \\
\hline 8 & 3.98 & 33.37 & 46 & 34 & 0.968 & 1781.17 \\
\hline
\end{tabular}

TABLE 5: Solution for varying $R-L-C$.

\begin{tabular}{lccccccc}
\hline$m$ & $T$ & $T_{1}$ & $L$ & $C$ & $R$ & $\theta$ & 0.962 \\
\hline 1 & 3.68 & 5.06 & 11.80 & 0.009 & 69.05 & 403.74 \\
2 & 3.99 & 9.47 & 16.92 & 0.026 & 49.69 & 0.968 & 421.76 \\
3 & 4.66 & 15.72 & 21.85 & 0.045 & 43.04 & 0.977 \\
5 & 4.58 & 24.59 & 34.03 & 0.080 & 40.19 & 0.976 & 538.86 \\
6 & 4.45 & 28.34 & 34.71 & 0.113 & 34.12 & 0.974 & 874.99 \\
7 & 4.29 & 31.61 & 0.007 & 717.11 & 0.006 & 0.972 \\
8 & 4.62 & 38.68 & 45.15 & 0.15 & 33.33 & 0.976 & 531.72 \\
9 & 4.58 & 42.92 & 45.10 & 0.20 & 29.59 & 0.976 & 620.29 \\
\hline
\end{tabular}

TABLE 6: Sensitivity analysis for various parameters.

\begin{tabular}{|c|c|c|c|c|c|c|c|c|}
\hline Parameter & $\%$ change & $m$ & $T$ & $T_{1}$ & $L(\%)$ & $R(\%)$ & $\theta$ & $Z$ \\
\hline \multirow{4}{*}{$h$} & +50 & 4 & 1 & 4.38 & 0.0 & 0.0 & 0.003 & 45.71 \\
\hline & +30 & 4 & 4.71 & 20.62 & 12 & 17 & 0.977 & 1830.14 \\
\hline & -30 & 1 & 3.46 & 4.76 & 0.7 & 4 & 0.957 & 966.53 \\
\hline & -50 & 13 & 1 & 13.38 & 0.0 & 0.0 & 0.001 & 18.75 \\
\hline \multirow{4}{*}{$c_{0}$} & +50 & 2 & 4.79 & 11.38 & 4 & 10 & 0.978 & 1776.64 \\
\hline & +30 & 4 & 4.44 & 19.42 & 12 & 17 & 0.974 & 1493.88 \\
\hline & -30 & 2 & 4.44 & 10.54 & 3 & 9 & 0.974 & 1311.98 \\
\hline & -50 & 10 & 1 & 13.38 & 0.0 & 0.0 & 0.004 & 23.84 \\
\hline \multirow{4}{*}{$q_{0}$} & +50 & 19 & 1 & 19.38 & 0 & 0 & 0.008 & 10.32 \\
\hline & +30 & 19 & 1 & 19.38 & 0 & 0 & 0.002 & 10.32 \\
\hline & -30 & 10 & 1 & 10.38 & 0 & 0 & 0.004 & 19.43 \\
\hline & -50 & 6 & 5.27 & 33.62 & 26 & 26 & 0.982 & 665.57 \\
\hline \multirow{4}{*}{ C } & +50 & 7 & 1 & 12.38 & 0 & 0 & 0.008 & 27.12 \\
\hline & +30 & 7 & 1 & 8.38 & 0 & 0 & 0.0004 & 27.12 \\
\hline & -30 & 19 & 1 & 4.38 & 0 & 0 & 0.00 & 10.93 \\
\hline & -50 & 17 & 4.91 & 85.3 & 432 & 147 & 0.979 & 216.45 \\
\hline \multirow{4}{*}{$b$} & +50 & 12 & 1 & 12.38 & 0 & 0 & 0.0002 & 24.24 \\
\hline & +30 & 12 & 1 & 12.38 & 0 & 0 & 0.0002 & 21.00 \\
\hline & -30 & 12 & 1 & 12.38 & 0 & 0 & 0.0002 & 11.31 \\
\hline & -50 & 8 & 4.55 & 38.14 & 47 & 34 & 0.976 & 1563.89 \\
\hline
\end{tabular}

TABLE 7: Postsensitivity average optimum value.

\begin{tabular}{lcccccc}
\hline & $\bar{h}$ & $\overline{c_{0}}$ & $\overline{q_{0}}$ & $\bar{C}$ & $\bar{b}$ & Model min. \\
$\bar{L}$ & 3.18 & 4.75 & 6.5 & 108 & 11.75 & 0.12 \\
$\bar{R}$ & 5.25 & 9.00 & 6.5 & 36.75 & 8.5 & 0.08 \\
$\overline{T_{1}}$ & 10.79 & 13.68 & 20.69 & 27.61 & 18.82 & 6.38 \\
$\bar{Z}$ & 715.30 & 1151.6 & 176.4 & 320.40 & 405.11 & 355.43 \\
\hline
\end{tabular}


Using Section 3.2, we have

$$
U_{i}=\alpha_{1} l^{2}+\alpha_{2} l t+\alpha_{3} t^{2}+\alpha_{4} l+\alpha_{5} t+\alpha_{6},
$$

and using (6), the sample matrix is

$$
M=\left[\begin{array}{cccccc}
10.11 & 34.31 & 116.42 & 3.18 & 10.79 & 1 \\
22.56 & 64.98 & 187.14 & 4.75 & 13.68 & 1 \\
42.25 & 134.49 & 428.08 & 6.5 & 20.69 & 1 \\
11664 & 2981.88 & 762.31 & 108 & 27.61 & 1 \\
138.06 & 221.14 & 354.19 & 11.75 & 18.82 & 1 \\
.0144 & .766 & 40.70 & .12 & 6.38 & 1
\end{array}\right]
$$

and the submatrices are as follows:

$$
\begin{aligned}
& M_{1}=\left[\begin{array}{cccccc}
l^{2} & l t & t^{2} & l & t & 1 \\
22.56 & 64.98 & 187.14 & 4.75 & 13.68 & 1 \\
42.25 & 134.49 & 428.08 & 6.5 & 20.69 & 1 \\
11664 & 2981.88 & 762.31 & 108 & 27.61 & 1 \\
138.06 & 221.14 & 354.19 & 11.75 & 18.82 & 1 \\
.0144 & .766 & 40.70 & .12 & 6.38 & 1
\end{array}\right], \\
& M_{2}=\left[\begin{array}{cccccc}
10.11 & 34.31 & 116.42 & 3.18 & 10.79 & 1 \\
l^{2} & l t & t^{2} & l & t & 1 \\
42.25 & 134.49 & 428.08 & 6.5 & 20.69 & 1 \\
11664 & 2981.88 & 762.31 & 108 & 27.61 & 1 \\
138.06 & 221.14 & 354.19 & 11.75 & 18.82 & 1 \\
.0144 & .766 & 40.70 & .12 & 6.38 & 1
\end{array}\right], \\
& M_{3}=\left[\begin{array}{cccccc}
10.11 & 34.31 & 116.42 & 3.18 & 10.79 & 1 \\
22.56 & 64.98 & 187.14 & 4.75 & 13.68 & 1 \\
l^{2} & l t & t^{2} & l & t & 1 \\
11664 & 2981.88 & 762.31 & 108 & 27.61 & 1 \\
138.06 & 221.14 & 354.19 & 11.75 & 18.82 & 1 \\
.0144 & .766 & 40.70 & .12 & 6.38 & 1
\end{array}\right] \\
& M_{4}=\left[\begin{array}{cccccc}
10.11 & 34.31 & 116.42 & 3.18 & 10.79 & 1 \\
22.56 & 64.98 & 187.14 & 4.75 & 13.68 & 1 \\
42.25 & 134.49 & 428.08 & 6.5 & 20.69 & 1 \\
l^{2} & l t & t^{2} & l & t & 1 \\
138.06 & 221.14 & 354.19 & 11.75 & 18.82 & 1 \\
.0144 & .766 & 40.70 & .12 & 6.38 & 1
\end{array}\right] \text {, } \\
& M_{5}=\left[\begin{array}{cccccc}
10.11 & 34.31 & 116.42 & 3.18 & 10.79 & 1 \\
22.56 & 64.98 & 187.14 & 4.75 & 13.68 & 1 \\
42.25 & 134.49 & 428.08 & 6.5 & 20.69 & 1 \\
11664 & 2981.88 & 762.31 & 108 & 27.61 & 1 \\
l^{2} & l t & t^{2} & l & t & 1 \\
.0144 & .766 & 40.70 & .12 & 6.38 & 1
\end{array}\right] \text {, } \\
& M_{6}=\left[\begin{array}{cccccc}
10.11 & 34.31 & 116.42 & 3.18 & 10.79 & 1 \\
22.56 & 64.98 & 187.14 & 4.75 & 13.68 & 1 \\
42.25 & 134.49 & 428.08 & 6.5 & 20.69 & 1 \\
11664 & 2981.88 & 762.31 & 108 & 27.61 & 1 \\
138.06 & 221.14 & 354.19 & 11.75 & 18.82 & 1 \\
l^{2} & l t & t^{2} & l & t & 1
\end{array}\right] \text {. }
\end{aligned}
$$

Then, the corresponding determinants are

$$
\begin{aligned}
& \Delta=|M|=9.79 \times 10^{7}, \\
& \Delta_{1}=\left|M_{1}\right|=2.55 \times 10^{9}+6.96 \times 10^{8} l-3.88 \times 10^{7} l t \\
&-5.67 \times 10^{8} t+3.14 \times 10^{6} l^{2}+2.18 \times 10^{7} t^{2}, \\
& \Delta_{2}=\left|M_{2}\right|=-2.5 \times 10^{9}-6.06 \times 10^{8} l+3.33 \times 10^{7} l t \\
&+5.32 \times 10^{8} t-2.57 \times 10^{6} l^{2}-2.09 \times 10^{7} t^{2}, \\
& \Delta_{3}=\left|M_{3}\right|= 3.10 \times 10^{8}+7.03 \times 10^{7} l-4.65 \times 10^{6} l t \\
&-7.00 \times 10^{7} t+466677 l^{2}+3.24 \times 10^{6} t^{2}, \\
& \Delta_{4}=\left|M_{4}\right|=-348718.0-21282.3 l-9205.02 l t \\
&+53845.7 t+10819.3 l^{2}+359.41 t^{2}, \\
& \Delta_{5}=\left|M_{5}\right|= 1.56 \times 10^{8}+2.85 \times 10^{7} l-393365 l t \\
&-3.00 \times 10^{7} t-157185 l^{2}+792799 t^{2}, \\
& \Delta_{6}=\left|M_{6}\right|=-4.17 \times 10^{8}-1.88 \times 10^{8} l+1.06 \\
& \times 1 t+1.15 \times 10^{8} t-883222 l^{2}-4.96 t^{2} .
\end{aligned}
$$

Therefore, we have

$$
\begin{aligned}
U= & U_{1} \frac{\Delta_{1}}{\Delta}+U_{2} \frac{\Delta_{2}}{\Delta}+U_{3} \frac{\Delta_{3}}{\Delta}+U_{4} \frac{\Delta_{4}}{\Delta}+U_{5} \frac{\Delta_{5}}{\Delta}+U_{6} \frac{\Delta_{6}}{\Delta} \\
= & -11092.6-2471.77 l+2429.69 t+133.18 l t \\
& -10.27 l^{2}-95.49 t^{2} .
\end{aligned}
$$

Similarly, we have for thedata sets $\left(\bar{R}, \overline{T_{1}}, \bar{Z}\right)$ that

$$
V_{i}=\beta_{1} r^{2}+\beta_{2} r t+\beta_{3} t^{2}+\beta_{4} r+\beta_{5} t+\beta_{6} .
$$

Then, the sample matrix is

$$
N=\left[\begin{array}{cccccc}
27.56 & 56.62 & 116.42 & 5.25 & 10.79 & 1 \\
81 & 123.12 & 187.14 & 9 & 13.68 & 1 \\
42.25 & 134.49 & 428.08 & 6.5 & 20.69 & 1 \\
1350.56 & 1014.67 & 762.31 & 36.75 & 27.61 & 1 \\
72.25 & 159.97 & 354.19 & 8.5 & 18.82 & 1 \\
.0064 & .5104 & 40.70 & .08 & 6.38 & 1
\end{array}\right]
$$


and the submatrices are as follows:

$$
\begin{aligned}
& N_{1}=\left[\begin{array}{cccccc}
r^{2} & r t & t^{2} & r & t & 1 \\
81 & 123.12 & 187.14 & 9 & 13.68 & 1 \\
42.25 & 134.49 & 428.08 & 6.5 & 20.69 & 1 \\
1350.56 & 1014.67 & 762.31 & 36.75 & 27.61 & 1 \\
72.25 & 159.97 & 354.19 & 8.5 & 18.82 & 1 \\
.0064 & .5104 & 40.70 & .08 & 6.38 & 1
\end{array}\right], \\
& N_{2}=\left[\begin{array}{cccccc}
27.56 & 56.62 & 116.42 & 5.25 & 10.79 & 1 \\
r^{2} & r t & t^{2} & r & t & 1 \\
42.25 & 134.49 & 428.08 & 6.5 & 20.69 & 1 \\
1350.56 & 1014.67 & 762.31 & 36.75 & 27.61 & 1 \\
72.25 & 159.97 & 354.19 & 8.5 & 18.82 & 1 \\
.0064 & .5104 & 40.70 & .08 & 6.38 & 1
\end{array}\right] \text {, } \\
& N_{3}=\left[\begin{array}{cccccc}
27.56 & 56.62 & 116.42 & 5.25 & 10.79 & 1 \\
81 & 123.12 & 187.14 & 9 & 13.68 & 1 \\
r^{2} & r t & t^{2} & r & t & 1 \\
1350.56 & 1014.67 & 762.31 & 36.75 & 27.61 & 1 \\
72.25 & 159.97 & 354.19 & 8.5 & 18.82 & 1 \\
.0064 & .5104 & 40.70 & .08 & 6.38 & 1
\end{array}\right] \text {, }
\end{aligned}
$$$$
N_{4}=\left[\begin{array}{cccccc}
27.56 & 56.62 & 116.42 & 5.25 & 10.79 & 1 \\
81 & 123.12 & 187.14 & 9 & 13.68 & 1 \\
42.25 & 134.49 & 428.08 & 6.5 & 20.69 & 1 \\
r^{2} & r t & t^{2} & r & t & 1 \\
72.25 & 159.97 & 354.19 & 8.5 & 18.82 & 1 \\
.0064 & .5104 & 40.70 & .08 & 6.38 & 1
\end{array}\right] \text {, }
$$$$
N_{5}=\left[\begin{array}{cccccc}
27.56 & 56.62 & 116.42 & 5.25 & 10.79 & 1 \\
81 & 123.12 & 187.14 & 9 & 13.68 & 1 \\
42.25 & 134.49 & 428.08 & 6.5 & 20.69 & 1 \\
1350.56 & 1014.67 & 762.31 & 36.75 & 27.61 & 1 \\
r^{2} & r t & t^{2} & r & t & 1 \\
.0064 & .5104 & 40.70 & .08 & 6.38 & 1
\end{array}\right] \text {, }
$$$$
N_{6}=\left[\begin{array}{cccccc}
27.56 & 56.62 & 116.42 & 5.25 & 10.79 & 1 \\
81 & 123.12 & 187.14 & 9 & 13.68 & 1 \\
42.25 & 134.49 & 428.08 & 6.5 & 20.69 & 1 \\
1350.56 & 1014.67 & 762.31 & 36.75 & 27.61 & 1 \\
72.25 & 159.97 & 354.19 & 8.5 & 18.82 & 1 \\
r^{2} & r t & t^{2} & r & t & 1
\end{array}\right] \text {. }
$$

Now, the corresponding determinants are

$$
\begin{aligned}
\nabla=|N|= & -7.27 \times 10^{6}, \\
\nabla_{1}=\left|N_{1}\right|= & 1.64 \times 10^{8}+1.35 \times 10^{7} r-21280.9 r t \\
& -3.23 \times 10^{7} t-387783 r^{2}+1.02 \times 10^{6} t^{2}, \\
\nabla_{2}=\left|N_{2}\right|= & -1.34 \times 10^{8}-1.43 \times 10^{7} r+293184 r t \\
& +2.67 \times 10^{7} t+215282 r^{2}-873582 t^{2},
\end{aligned}
$$

TABLE 8: Optimal solution for MLI.

\begin{tabular}{lccc}
\hline $\bar{L}(\%)$ & $\bar{R}(\%)$ & $\overline{T_{1}}$ (days) & $\bar{Z}(\$)$ \\
\hline$[0.86,2.12]$ & {$[4.80,7.52]$} & {$[8.71,12.0]$} & {$[2878.99,3551.95]$} \\
\hline
\end{tabular}

$$
\begin{aligned}
\nabla_{3}=\left|N_{3}\right|= & -2.40 \times 10^{7}-4.16 \times 10^{6} r+362704 r t \\
& +5.49 \times 10^{6} t-103105 r^{2}-266769 t^{2}
\end{aligned}
$$$$
\nabla_{4}=\left|N_{4}\right|=2.93 \times 10^{6}+289556 r+2888.14 r t
$$$$
-570838 t-15514.3 r^{2}+16981.2 t^{2}
$$$$
\nabla_{5}=\left|N_{5}\right|=4.68 \times 10^{7}+7.89 \times 10^{6} r-586656 r t
$$$$
-1.013 \times 10^{7} t+156218 r^{2}+429368 t^{2}
$$$$
\nabla_{6}=\left|N_{6}\right|=-6.29 \times 10^{7}-3.25 \times 10^{6} r-50838.9 r t
$$$$
+1.08 \times 10^{7} t+134901 r^{2}-324766 t^{2} .
$$

Therefore, we have

$$
\begin{aligned}
V= & V_{1} \frac{\nabla_{1}}{\nabla}+V_{2} \frac{\nabla_{2}}{\nabla}+V_{3} \frac{\nabla_{3}}{\nabla}+V_{4} \frac{\nabla_{4}}{\nabla}+V_{5} \frac{\nabla_{5}}{\nabla}+V_{6} \frac{\nabla_{6}}{\nabla} \\
= & 6009.91+744.23 r-1124.29 t-18.098 r t \\
& -8.06 r^{2}+35.69 t^{2} .
\end{aligned}
$$

Here, considering average conducting factor $C=15$, our problem reduces to

$$
\text { Maximize } \begin{aligned}
U= & -11092.6-2471.77 l+2429.69 t \\
& +133.18 l t-10.27 l^{2}-95.49 t^{2}, \\
V= & 6009.91+744.23 r-1124.29 t-18.098 r t \\
& -8.06 r^{2}+35.69 t^{2}
\end{aligned}
$$

Subject to $R^{2} C<4 L$.

To solve the above, we will maximize $U$ and $V$ separately subject to the given condition $R^{2} C<4 L$ and obtain the result in Table 8.

5.2. Interpretation on Optimal Values in Table 8. We have constructed an MLI function to make a significant decision over the model, so we need not discuss the other tables. From Table 8 , we see that the range of the average cost is [\$2878.99, \$3551.95], for the range of induced factor [0.86\%, $2.12 \%]$, the range of resisting index is [4.80\%, 7.52\%], and all quantity exhausted time range is [8.71 days, 12.00 days]. This information can be helpful enough for a decision maker of a global trading system.

5.3. Graphical Interpretation of the Model. From Figures 3 and 4 , it is observed that the average minimum cost is nominal 


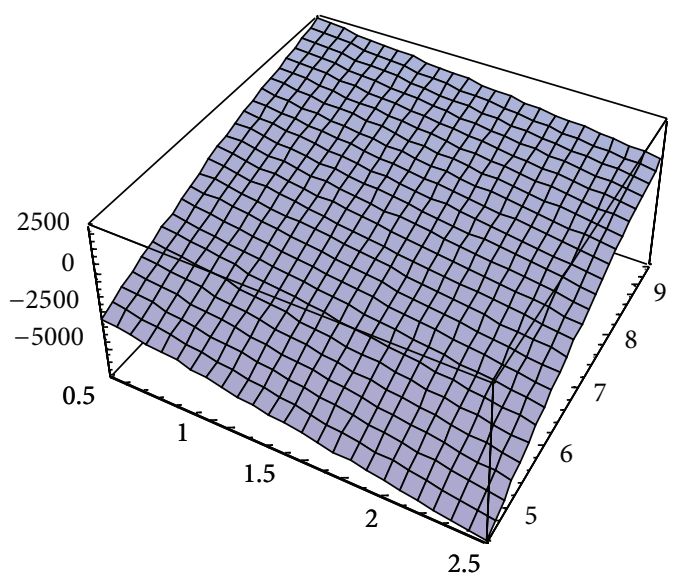

Figure 3: Cost model for varying $L-T$.

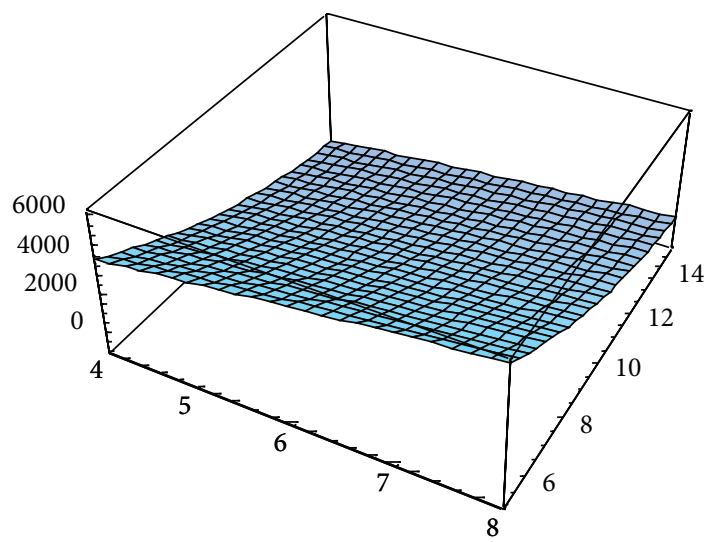

FIgURE 4: Cost model for varying $R-T$.

challenge from other setups. Breaking this myth, we have observed in a bird's view that the present time is actually of "sleep with challenge and awake to challenge others" or "hire and fire." The proposed model helps the industrial engineers in knowing how to control the inventory model under several limitations which are newly introduced in the inventory literature.

The basic features of our model are as follows.

(1) Peoples' motivations result in induced factor form generation, means demand rate becomes oscillatory in nature that resulting the inventory circuit as discharging of a condenser in an electrical circuit.

(2) Key of the inventory chain indicates whether the shop is open or closed. No induced factor will come if the shop is closed, but it will take little time (phase time difference) to generate a considerable induced factor.

(3) As the inventory is depicted with time, the induced factor began to die out and its presence will disappear in the first shift at the end of cycle time which is a common phenomenon of any kind of physical system.

(4) Frequency of the oscillatory inventory curve generates phases, namely, first shift for a day time and the second shift for the night or other similar means.

(5) The definitions like core customers, surface customers, and induced and conducting factors have been discussed under competitive sphere of market and investments.

(6) Numerical multivariate interpolation has been utilized to overcome any kind of discrepancies over the decision variables of the model.

(Figure 3) whenever we take the induced factor around 2.5\% and the exhaust time is nearer to 5 days for the constant values of the resisting index $R$ and conducting factor $C$. Moreover, the low induced factor (around 0.5\%) and long exhausting time cause a high cost when the $R$ and $C$ values are kept constant.

Figure 4 shows that the average minimum cost occurs at around $4 \%$ resisting index and the inventory level is empty within 14 days for constant values of the conducting factor $C$ and induced factor $L$. The average cost is around $\$ 6000$, whenever the resisting index $R$ lies around $8 \%$ near 5-day exhausting time for the fixed values of $L$ and $C$. Analyzing both the figures, we may conclude that Figure 3 can give more economic result than the other.

\section{Conclusion}

In oligopolistic marketing system, competitions among the companies/industries are very much tuff. In these situations, it is hardly possible to survive and sustain a company under globalized trading system. The traditional concept was that the company will build inventory setup to provide customers' needs under smooth and nice path without getting any

The major limitations of the model are as follows.

(1) The model is of multivalued decision variables, so no finite search direction will be observed. For this reason we have to rely on some line search technique also.

(2) In many cases the relational of equations among the variables $R, L$, and $C$ may not effect remarkably on the cost optimization model.

(3) Several definitions may not support the so-called "inventory management problems." Therefore, our model of study represents a special category of EOQ model, and we rename it as phase inventory model.

\section{Scope of Future Work}

Anybody may link up the inventory process with the trade economics, thermodynamic process, and the electrical circuit theory in a new outlook also. A concept of steady and equilibrium production and supply system can be chalked out from this paper. 


\section{Appendix}

(1) The time distance for a complete oscillation (wave length) is calculated as follows:

$$
\begin{gathered}
\delta=t_{3}-t_{1}=t_{5}-t_{3}=t_{7}-t_{5}=\cdots=t_{2 m+1}-t_{2 m-1} \\
\Longrightarrow m \delta=t_{2 m+1}-t_{1}=\frac{(2 m+1) \pi-\varphi}{w}-\frac{\pi-\varphi}{w}=\frac{2 m \pi}{w} \\
\Longrightarrow \delta=\frac{2 \pi}{w}=\frac{2 \pi}{w_{0} \sqrt{1-\theta^{2}}}=\frac{1}{\sqrt{1-\theta^{2}}}=T \\
\left(\therefore w_{0}=2 \pi, \text { by Definition } 6\right) .
\end{gathered}
$$

Again, we have $\varphi=2 \pi x / \delta=2 \pi x \sqrt{1-\theta^{2}}=w x$, where $x$ and $\delta$ are measured in time units.

(2) To discuss the dimension of both sides of the following equation:

$$
\frac{d^{2} q}{d t^{2}}+2 \theta w_{0} \frac{d q}{d t}+w_{0}^{2} q=0
$$

we take

$$
\begin{gathered}
{[q]=\text { unit }=u, \quad\left[w_{0}\right]=\frac{\text { Number }}{\text { Time }}=t^{-1},} \\
{\left[\frac{d q}{d t}\right]=u \times t^{-1}, \quad\left[\frac{d^{2} q}{d t^{2}}\right]=u \times t^{-2},} \\
{[\theta]=\text { number, } \quad\left[w_{0}^{2} q\right]=u \times t^{-2} .}
\end{gathered}
$$

So,

$$
\left[2 \theta w_{0} \frac{d q}{d t}\right]=t^{-1} \times u \times t^{-1}=u \times t^{-2} .
$$

(3) Using (12), the simplified form of (17) is obtained as follows:

$$
\begin{aligned}
& \int q(t) d t= \frac{q_{0}}{\sqrt{1-\theta^{2}}} \int e^{-2 \pi \theta t} \operatorname{Sin} w(t+x) d t \\
&=-\frac{q_{0} e^{-2 \pi \theta t}}{2 \pi \sqrt{1-\theta^{2}}}[\theta \operatorname{Sin} w(t+x)\left.\quad+\sqrt{1-\theta^{2}} \operatorname{Cos} w(t+x)\right] \\
&=-\frac{q_{0} e^{-2 \pi \theta t}}{w} \operatorname{Sin} w(t+2 x) .
\end{aligned}
$$

Therefore,

$$
\begin{array}{rl}
\int_{0}^{\pi / w-x} & q(t) d t \\
= & -\frac{q_{0}}{w}\left[\operatorname{Sin}(\pi+w x) e^{2 \pi \theta x-2 \theta \pi^{2} / w}-\operatorname{Sin}(2 w x)\right] \\
& =\frac{q_{0}}{2 \pi}\left[e^{2 \pi \theta x-2 \theta \pi^{2} / w}+2 \theta\right], \\
\sum_{r=1}^{m} \int_{2 r \pi / w-x}^{(2 r+1) \pi / w-x} q(t) d t & \quad \times e^{2 \pi \theta x-2 \theta \pi^{2} / w-4 \theta \pi^{2} r / w} \\
= & -\frac{q_{0}}{w} \sum_{r=1}^{m}[\operatorname{Sin}\{(2 r+1) \pi+w x\} \\
= & \frac{q_{0} e^{2 \pi \theta x-2 \theta \pi^{2} / w}\left(1-e^{-4 \theta \pi^{2} m / w}\right)}{2 \pi} \frac{\left(e^{2 \theta \pi^{2} / w}-1\right)}{\left.(2 r \pi+w x) e^{2 \pi \theta x-4 \theta \pi^{2} r / w}\right]} \\
= & \frac{q_{0} \operatorname{Sin} w x e^{2 \pi \theta x}}{w}\left(1+e^{-2 \theta \pi^{2} / w}\right) \sum_{r=1}^{m}\left[e^{-4 \theta \pi^{2} r / w}\right] \\
& \left.\frac{q_{0} e^{2 \pi \theta x}}{2 \pi}\left(1+e^{-2 \theta \pi^{2} / w}\right) \frac{\left(1-e^{-4 \theta \pi^{2} m / w}\right)}{\left(e^{2} / w\right.}-1\right)
\end{array}
$$

(4) Using (12), the simplified form of (19) is obtained as follows:

$$
\begin{aligned}
\int \frac{d^{2} q}{d t^{2}} d t= & \frac{8 \pi^{3} q_{0}}{w} \int e^{-2 \pi \theta t} \operatorname{Sin} w(t-x) d t \\
= & -\frac{8 \pi^{3} q_{0} e^{-2 \pi \theta t}}{2 \pi w} \\
& \times\left[\theta \operatorname{Sin} w(t-x)+\sqrt{1-\theta^{2}} \operatorname{Cos} w(t-x)\right] \\
= & -\frac{4 \pi^{2} q_{0} e^{-2 \pi \theta t} \operatorname{Sin} w t}{w}
\end{aligned}
$$

Thus,

$$
\begin{array}{r}
c_{0} L \times \int \frac{d^{2} q}{d t^{2}} d t=-\frac{c_{0} q_{0} e^{-2 \pi \theta t}}{C w} \operatorname{Sin} w t, \\
{\left[\text { since, } L=\frac{1}{4 \pi^{2} C}\right] .}
\end{array}
$$


Now,

$$
\begin{aligned}
& \left(\sum_{r=0}^{m-1} \int_{2 r \pi / w+x}^{(2 r+1) \pi / w+x}-\sum_{r=1}^{m} \int_{(2 r-1) \pi / w+x}^{2 r \pi / w+x}\right) \\
& \times e^{-2 \pi \theta t} \operatorname{Sin} w(t-x) d t \\
& =\sum_{r=0}^{m-1}\left[\operatorname{Sin}\{(2 r+1) \pi+w x\} e^{-2 \pi \theta x-2 \theta \pi^{2} / w-4 \theta \pi^{2} r / w}\right. \\
& \left.-\operatorname{Sin}(2 r \pi+w x) e^{-2 \pi \theta x-4 \theta \pi^{2} r / w}\right] \\
& -\sum_{r=1}^{m}\left[\operatorname{Sin}\{(2 r+1) \pi+w x\} e^{-2 \pi \theta x-4 \theta \pi^{2} r / w}\right. \\
& \left.-\operatorname{Sin}((2 r-1) \pi+w x) e^{-2 \pi \theta x+2 \theta \pi^{2} / w-4 \theta \pi^{2} r / w}\right] \\
& =-e^{-2 \pi \theta x} \operatorname{Sin} w x\left(1+e^{-2 \theta \pi^{2} / w}\right) \\
& \times \sum_{r=0}^{m-1}\left[e^{-4 \theta \pi^{2} r / w}\right]-e^{-2 \pi \theta x} \operatorname{Sin} w x \\
& \times\left(1+e^{2 \theta \pi^{2} / w}\right) \sum_{r=1}^{m}\left[e^{-4 \theta \pi^{2} r / w}\right] \\
& =-e^{-2 \pi \theta x} \operatorname{Sin} w x\left[\left(1+e^{-2 \theta \pi^{2} / w}\right)\left(\frac{1-e^{-4 \theta \pi^{2} m / w}}{1-e^{-4 \theta \pi^{2} / w}}\right)\right. \\
& +e^{-4 \theta \pi^{2} / w}\left(1+e^{2 \theta \pi^{2} / w}\right) \\
& \left.\times\left(\frac{1-e^{-4 \theta \pi^{2} m / w}}{1-e^{-4 \theta \pi^{2} / w}}\right)\right] \\
& =-e^{-2 \pi \theta x} \operatorname{Sin} w x\left[\left(1+e^{2 \theta \pi^{2} / w}\right)\left(\frac{1-e^{-4 \theta \pi^{2} m / w}}{e^{2 \theta \pi^{2} / w}-1}\right)\right] .
\end{aligned}
$$

So,

$$
\begin{aligned}
c_{0} L & \times \int_{x}^{(2 m+1) \pi / w+x} \frac{d^{2} q}{d t^{2}} d t \\
& =\frac{c_{0} q_{0} e^{-2 \pi \theta x}}{2 \pi C}\left[\left(1+e^{2 \theta \pi^{2} / w}\right)\left(\frac{1-e^{-4 \theta \pi^{2} m / w}}{e^{2 \theta \pi^{2} / w}-1}\right)\right] .
\end{aligned}
$$

\section{Conflict of Interests}

The authors declare that there is no conflict of interests regarding the publication of this paper.

\section{References}

[1] F. W. Harris, "What quantity to make at once," in The Library of Factory Management, Operation and Costs, pp. 47-52, 1995.

[2] A. Andriolo, D. Battini, R. W. Grubbstrom, A. Persona, and F. Sgarbossa, "A century of evolution from Harriss basic lot size model; survey and research agenda," International Journal of Production Economics, vol. 155, pp. 16-38, 2014.

[3] G. Bruno, A. Genovese, and C. Piccolo, "The capacitated lot size model: a powerful tool for logistics decisión making," International Journal of Production Economics, vol. 155, pp. 380390, 2014.

[4] B. R. Sarker, "Consignment stocking policy models for supply chain systems: a critical review and comparative perspective," International Journal of Production Economics, vol. 155, pp. 5267, 2014.

[5] J.-T. Teng, C.-T. Chang, and S. K. Goyal, "Optimal pricing and ordering policy under permissible delay in payments," International Journal of Production Economics, vol. 97, no. 2, pp. 121-129, 2005.

[6] L. E. Cárdenas- Barrón, “Optimal ordering policies in response to a discount offer: extensions," International Journal of Production Economics, vol. 122, pp. 774-782, 2009.

[7] L. E. Cárdenas- Barrón, "Optimal ordering policies in response to a discount offer: corrections," International Journal of Production Economics, vol. 122, no. 2, pp. 783-789, 2009.

[8] L. E. Cárdenas- Barrón, "A complement to 'A comprehensive note on: AN economic order quantity with imperfect quality and quantity discounts," Applied Mathematical Modelling, vol. 36, no. 12, pp. 6338-6340, 2012.

[9] L. E. Cárdenas-Barrón, N. R. Smith, and S. K. Goyal, “Optimal order size to take advantage of a one-time discount offer with allowed backorders," Applied Mathematical Modelling, vol. 34, no. 6, pp. 1642-1652, 2010.

[10] S. K. Ghosh, S. Khanra, and K. S. Chaudhuri, "Optimal price and lot size determination for a perishable product under conditions of finite production, partial backordering and lost sale," Applied Mathematics and Computation, vol. 217, no. 13, pp. 6047-6053, 2011.

[11] B. Sarkar, S. Saren, and H.-M. Wee, "An inventory model with variable demand, component cost and selling price for deteriorating items," Economic Modelling, vol. 30, no. 1, pp. 306310, 2013.

[12] A. Bozorgi, J. Pazour, and D. Nazzal, "A new inventory model for cold items that considers costs and emissions," International Journal of Production Economics, vol. 155, pp. 114-125, 2014.

[13] M. S. Sodhi, N. S. Sodhi, and C. S. Tang, "An EOQ model for MRO customers under stochastic price to quantify bullwhip effect for the manufacturer," International Journal of Production Economics, vol. 155, pp. 132-142, 2014.

[14] Y. Yang, Q. Yuan, W. Xue, and Y. Zhou, "Analysis of batch ordering inventory models with setup cost and capacity constraint," International Journal of Production Economics, vol. 155, pp. 340350, 2014.

[15] L. C. Coelho and G. Laporte, "Improved solutions for inventory routing problems through valid inequalities and input ordering," International Journal of Production Economics, vol. 155, pp. 391-397, 2014.

[16] T. Avinadav, A. Herbon, and U. Spiegel, "Optimal ordering and pricing policy for demand functions that are separable into price and inventory age," International Journal of Production Economics, vol. 155, pp. 406-417, 2014.

[17] S. Chand and S. Sethi, "Multi-period lot-sizing with stationary demand: extension to forecast horizons," in Handbook of EOQ Inventory Problems, vol. 197 of International Series in Operations Research \& Management Science, pp. 23-42, Springer, New York, NY, USA, 2014. 
[18] B. B. Keskin and I. Capar, "The utility of EOQ in supply chain design and operation," International Series in Operations Research \& Management Science, vol. 197, pp. 221-245, 2014.

[19] A. Gambini, G. M. Scarpello, and D. Ritelli, "Existance of EOQ and its evaluation: some cases of stock blow down dynamics depending on its level," in Handbook of EOQ Inventory Problems, vol. 197 of International Series in Operations Research \& Management Science, pp. 59-78, 2014.

[20] R. J. Dolan, "Quantity discounts: managerial issues and research opportunities," Marketing Science, vol. 6, pp. 1-22, 1987.

[21] J. P. Monahan, "A quantity discount pricing model to increase vendor profits," Management Science, vol. 30, no. 6, pp. 720-726, 1984.

[22] D. S. Dave, K. E. Fitzpatrick, and J. R. Baker, "An advertisinginclusive production lot size model under continuous discount pricing," Computers and Industrial Engineering, vol. 30, no. 1, pp. 147-159, 1996.

[23] P. K. Tripathy and M. Pattanaik, "An entropic order quantity model with fuzzy holding cost and fuzzy disposal cost for perishable items under two component demand and discounted selling price," Pakistan Journal of Statistics and Operation Research, vol. 4, no. 2, pp. 93-110, 2008.

[24] J. K. Syed and L. A. Aziz, "An inventory model with price breaks: fuzzy approach," African Journal of Mathematics and Computer Science Research, vol. 3, pp. 232-236, 2010.

[25] L. R. Weatherford and S. E. Bodily, "A taxonomy and research overview of perishable asset revenue management, overbooking and pricing," Operations Research, vol. 40, pp. 831-844, 1992.

[26] J. Bryant, "A thermodynamic theory of economics," International Journal of Exergy, vol. 4, no. 3, pp. 302-337, 2007.

[27] E. Smith and D. K. Foley, "Classical thermodynamics and economic general equilibrium theory," Santa Fe Institute, 2004.

[28] F. Soddy, The Role of Money, Routledge, London, UK, 1934. 

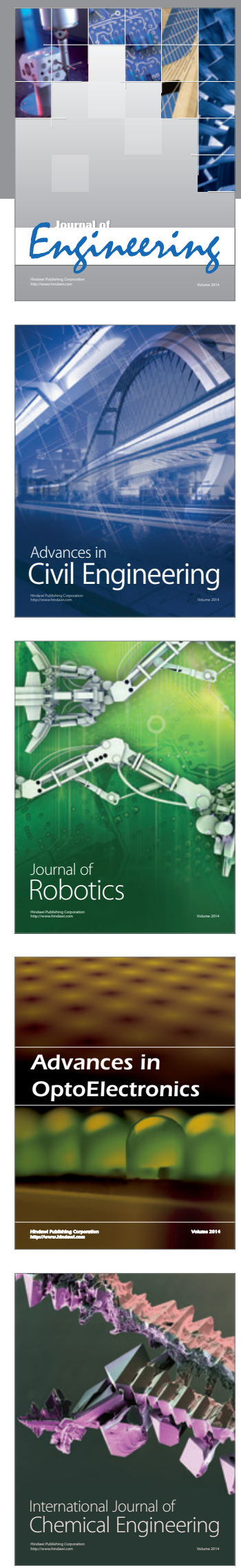

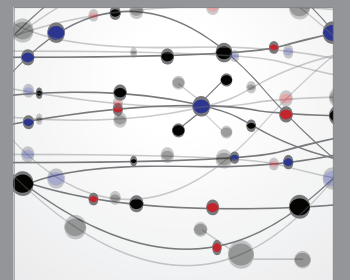

The Scientific World Journal
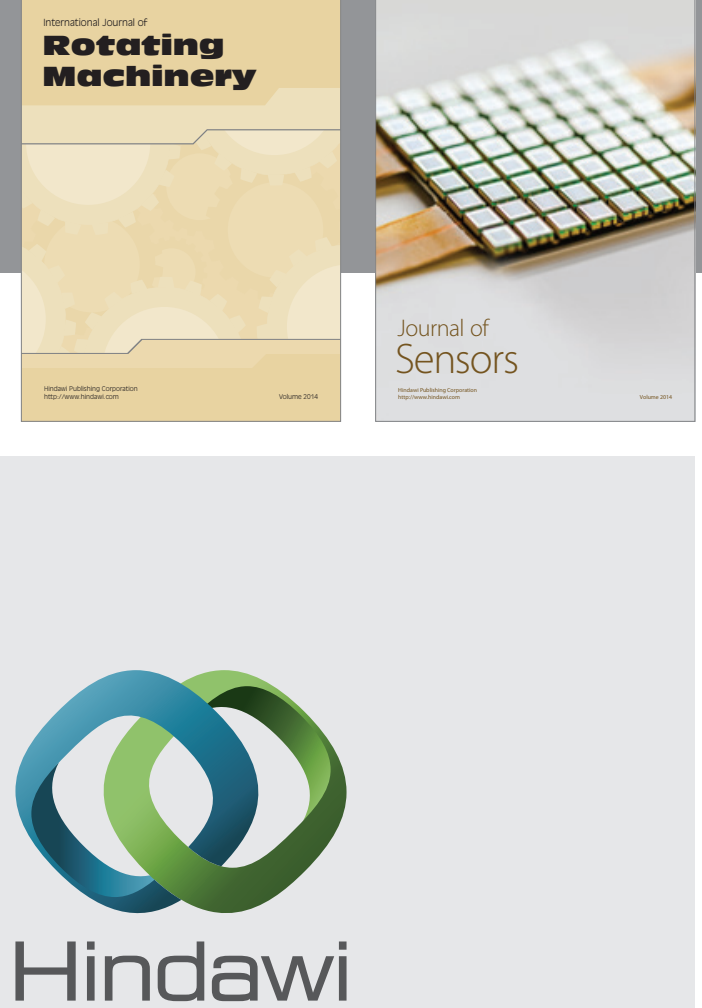

Submit your manuscripts at http://www.hindawi.com
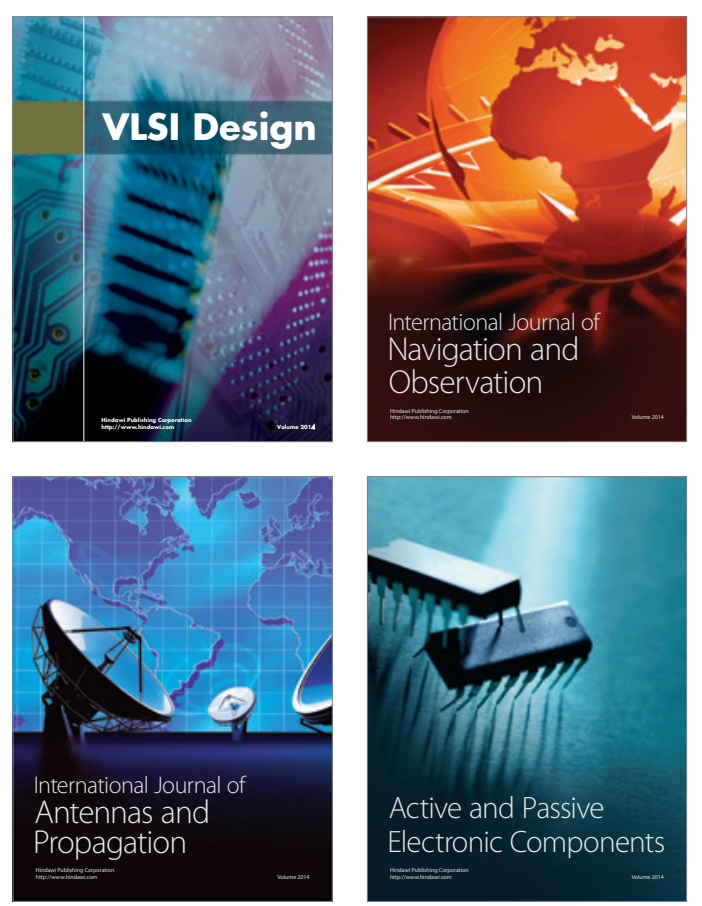
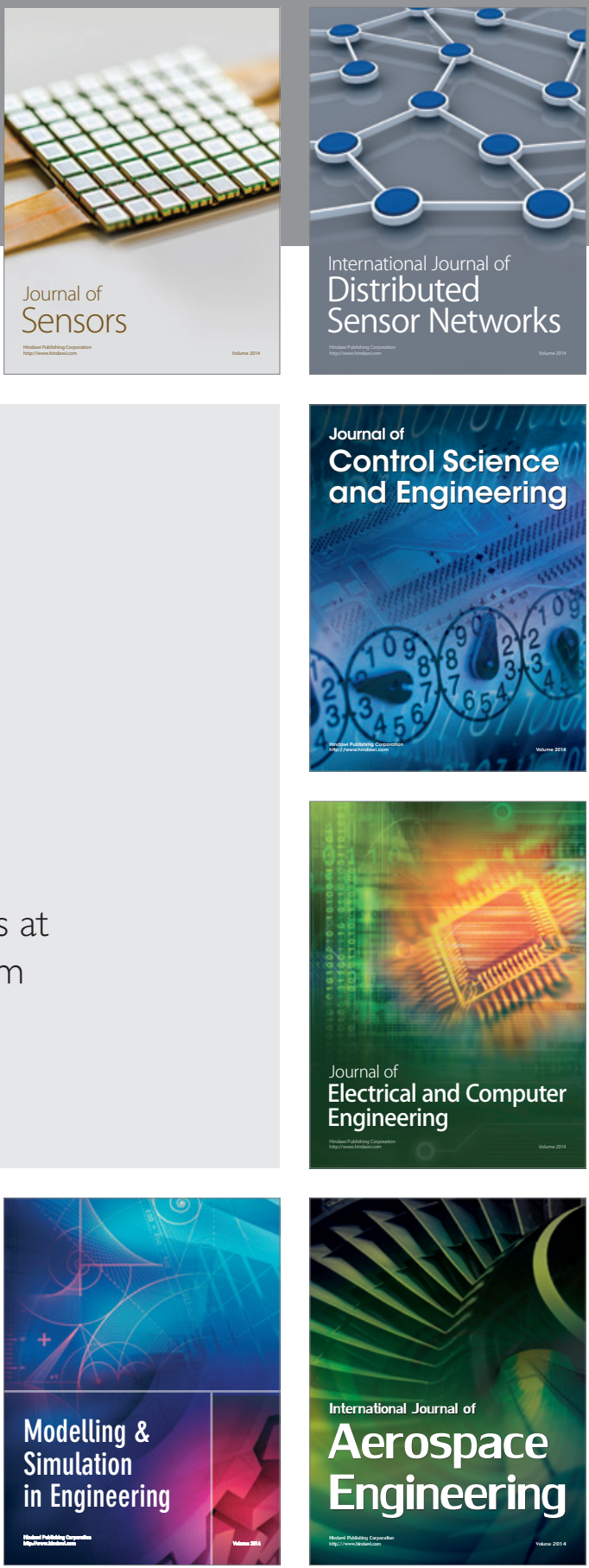

Journal of

Control Science

and Engineering
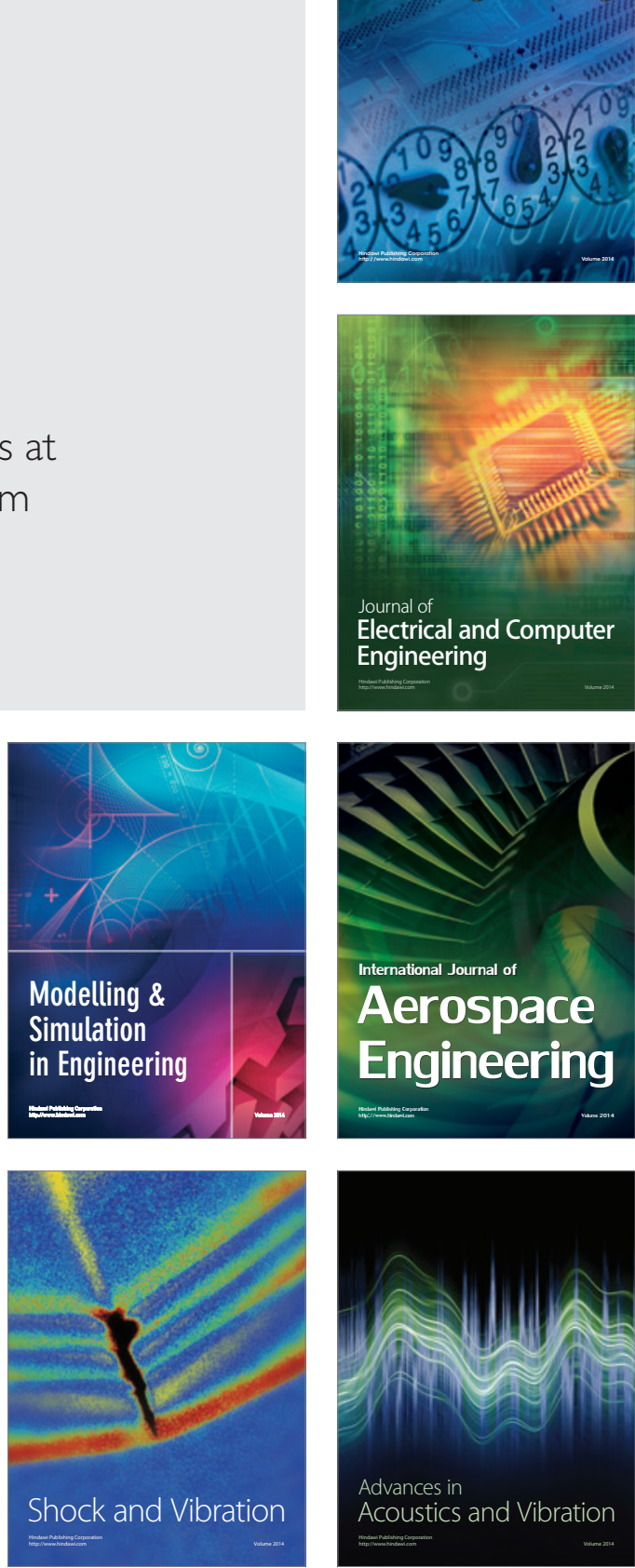